\title{
Prevalence of pulmonary embolism in patients with COVID-19 at the time of hospital admission
}

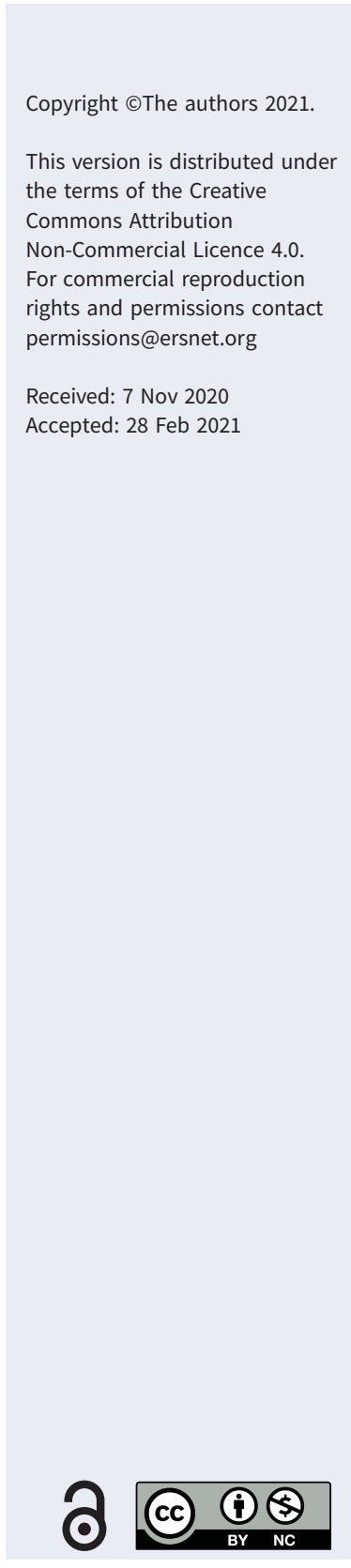

To the Editor:

A high prevalence of venous thromboembolism (VTE) has been reported during intensive care unit (ICU) hospitalisation in patients with severe coronavirus disease 2019 (COVID-19) [1, 2]. In most cases, the diagnosis of pulmonary embolism (PE) was incidental as patients underwent computed tomography pulmonary angiography (CTPA) for aggravation of their respiratory condition. Higher mortality is also described in patients with high D-dimer levels suggesting that VTE complication may contribute to unfavourable prognosis $[3,4]$. Even though, prevalence of thromboembolic complications during ICU hospitalisation seems to be high, the prevalence of pulmonary embolism at hospital admission for COVID-19 is unknown and may be underestimated.

In the present research letter, we report a prospective multicentre study that evaluates the pulmonary embolism prevalence in patients admitted for COVID-19, at the time of admission in three tertiary hospitals, Bicêtre Hospital and Béclère Hospital, University Paris-Saclay, and European Georges Pompidou Hospital, Paris University (Paris, France).

All consecutive adult outpatients hospitalised between April 15, 2020, to May 23, 2020, with a diagnosis of COVID-19 were included and underwent a CTPA, if not contraindicated, at the time of hospital admission. Patients with renal failure $\left(<30 \mathrm{~mL} \cdot \mathrm{min}^{-1}\right.$ of clearance) or contraindications to iodinated contrast material were excluded from the study. COVID-19 diagnosis was confirmed by the presence of positive Severe acute respiratory syndrome coronavirus 2 (SARS-CoV-2) RT-PCR and/or typical computed tomography abnormalities (i.e. ground-glass opacities and/or consolidation in the lung periphery) $[5,6]$. Hospital admission was decided by the clinician in charge at the emergency department of each participating hospital according to clinical criteria.

The computed tomography patterns of COVID-19 pneumonia and the presence of pulmonary embolism were analysed locally by a senior radiologist and a pulmonologist. Patients without pulmonary embolism received prophylactic anticoagulation during hospitalisation, according to local practice.

Patients were prospectively followed-up for three months or until death, by a telephone interview within 3 months after admission.

Performing systematic CTPA was in accordance with the multidisciplinary medical crisis team for COVID-19 and all patients received oral information about data collection. Upon consent, data were recorded in an anonymous database registered to the National Commission on Informatics and Liberty (n²6750045200441). The study was been approved by the CERAPHP Centre (Comité d'éthique de la recherche APHP Centre; IRB registration: \#00011928).

Quantitative data are expressed as median (interquartile range) and qualitative data are expressed as n (\%). The prevalence of pulmonary embolism was calculated, and the $95 \%$ confidence intervals were determined. Comparisons between groups were performed using the independent student's t-test if the distribution of variables was normal and by the Wilcoxon test if not normally distributed. A multivariable logistic regression model adjusted for age and sex was calculated for significant results $(\mathrm{p}<0.05)$. Categorical variables were 
compared using the Chi-squared test for independence. XLSTAT (Addinsoft, 2019, Long Island, NY, USA) and Graphpad prism (GraphPad Software, La Jolla, CA, USA) software were used. A p-value of $<0.05$ was considered statistically significant.

During the study period, 135 consecutives outpatients were hospitalised for COVID-19 at the three participating hospitals. Among them, 29 (21.5\%) were excluded from the study, mainly due to contraindications for iodinated contrast administration $(n=12)$, inability to understand oral information $(n=7)$ and screening failure $(n=10)$. Of the 106 included patients, five $(4.7 \%)$ patients were directly admitted to an ICU.

Overall, pulmonary embolism was diagnosed in 15 out of 106 patients giving a prevalence of 14.2\% (95\% CI 7.5-20.8). Among the five patients directly admitted in ICU, one had a confirmed pulmonary embolism at admission. Table 1 summarises the characteristics of included patients and the chest computed tomography scan features. CTPA was well tolerated with no aggravation of renal function $(n=87 ; p=0.52)$ and only one complication $(0.9 \%)$ was reported due to iodinated material extravasation with no severe consequences.

Patients with confirmed pulmonary embolism on admission had significantly higher D-dimer median concentrations, more often needed oxygen administration in the emergency department and had longer median time from symptoms to hospital admission as compared to patients without pulmonary embolism. In multivariable analysis, only high D-dimer median concentrations (OR 1.001 (1-1.002)) and time from symptoms to hospital admission (OR 1.103 (1.019-1.193)) were independently associated with pulmonary embolism.

No significant difference was observed for computed tomography staging of COVID-19 lung involvement between patients with and without pulmonary embolism $(\mathrm{p}=0.85)$ and five (33.3\%) out of 15 patients had pulmonary embolism in the same location as COVID-19 pneumonia. Pulmonary embolism was lobar in seven (46.7\%), segmental in six (40\%) and sub-segmental in two (13.3\%) cases, respectively. Right ventricular/left ventricular diameter ratio>1 was observed in five patients (33.3\%).

During the time of hospitalisation, 15 (16.5\%) out of 91 patients had a repeated CTPA for clinical worsening without identification of any new pulmonary embolisms. Patients without pulmonary embolism at admission received standard VTE prophylaxis by enoxaparin $4000 \mathrm{IU} \cdot \mathrm{day}^{-1}$. In ICU patients, obese patients (BMI>35) and patients with high level of biological inflammation (D-dimer level greater than $1000 \mu \mathrm{g} \cdot \mathrm{L}^{-1}$ ) high-intensity VTE prophylaxis by Enoxaparin $4000 \mathrm{IU} / 12 \mathrm{~h}$ was used (or enoxaparin $6000 \mathrm{IU} \cdot 12 \mathrm{~h}^{-1}$ in case of weight greater than $120 \mathrm{~kg}$ ). After discharge, standard VTE prophylaxis was

TABLE 1 Baseline characteristics and comparison between patients with pulmonary embolism and patients without pulmonary embolism

\begin{tabular}{|c|c|c|c|c|}
\hline & All patients & $\begin{array}{l}\text { Patients with } \\
\text { pulmonary embolism }\end{array}$ & $\begin{array}{c}\text { Patients without } \\
\text { pulmonary embolism }\end{array}$ & p-value \\
\hline Patients $\mathrm{n}$ & 106 & 15 & 91 & \\
\hline Age years & $63(53-82)$ & $80.5(53-89)$ & $63(52-80)$ & 0.09 \\
\hline Male & 48 (45) & $8(53)$ & $40(44)$ & 0.24 \\
\hline Body mass index & $27(21.5-32)(n=57)$ & $32(23.5-40)(n=8)$ & $25(21-30)(n=49)$ & 0.39 \\
\hline Time from COVID symptoms to hospital admission days & $7(3-15)$ & $14(8-22)$ & $7(3-15)$ & 0.01 \\
\hline Patients with risk factors for VTE & $32(30)$ & $8(53)$ & $26(29)$ & 0.10 \\
\hline Malignancy/history of malignancy & $16(15)$ & $2(13)$ & $14(15)$ & \\
\hline Immobilisation/surgery in the past 4 weeks & $12(11)$ & $2(13)$ & $12(13)$ & \\
\hline History of VTE & $8(7)$ & $3(20)$ & $5(5)$ & \\
\hline Oestrogen & $2(2)$ & $1(6)$ & $1(1)$ & \\
\hline D-dimer concentration ${ }^{\#} \mathrm{ng} \cdot \mathrm{mL}^{-1}$ & $1190(669-2245)(n=98)$ & $3220(2317-3855)(n=13)$ & $1047(620-1764)(n=85)$ & 0.001 \\
\hline Oxygen needed on admission & $33(31)$ & $9(60)$ & $24(26)$ & 0.03 \\
\hline \multicolumn{5}{|l|}{ CT of chest features of COVID-19 pneumonia } \\
\hline Mild $(<10 \%)$ and moderate $(10-25 \%)$ & $72(68)$ & $10(67)$ & $62(68)$ & 0.85 \\
\hline Severe $(25-50 \%)$, very severe $(50-75 \%)$ and critical $(>75 \%)$ & $34(32)$ & $5(33)$ & $29(32)$ & \\
\hline
\end{tabular}

Data are presented as median (interquartile range) or $\mathrm{n}(\%)$, unless otherwise stated. VTE: venous thromboembolism; COVID-19: coronavirus disease 2019. ": D-dimers plasma levels were obtained using either VIDAS D-dimer Exclusion, Biomerieux or STA-LIATEST, Diagnostica Stago. 
prolonged for 2-4 weeks in cases of persistently impaired mobility or persistently high levels of biological inflammation. 14 (13.2\%) out of 106 patients died during hospitalisation. Death was related to COVID-19 in 13 (93\%) cases and related to pulmonary embolism in only one (7\%) case. The median (interquartile range) interval between admission and death was 11 days (6-29). Patients who died were significantly older (median 83 years $(79.5-89.5)(p=0.001)$ ) and more of them had severe, very severe or critical features of COVID-19 pneumonia on CTPA $(\mathrm{p}=<0.001)$ at admission (table 1$)$.

Hospital mortality in patients with confirmed pulmonary embolism on admission was four (26.6\%) out of 15 and was not statistically different compared to hospital mortality of the group without pulmonary embolism $10(11.0 \%)$ out of 91 ( $p=0.09)$, which might be primarily driven by the small study size. At 3 months, no patients were lost to follow-up and seven additional deaths occurred for a total mortality rate of $21(19.8 \%)$ out of 106. Of these seven deaths, six were due to comorbidities and one was of unknown cause in an 85-year-old woman. No VTE recurrence occurred after hospital discharge in survivors.

This present study showed a prevalence of pulmonary embolism of 14.2\% (95\% CI 7.5-20.8) at the time of hospital admission for COVID-19. In line with previous reports, our study suggests that pulmonary embolism is a common complication among COVID-19 patients [1, 2]. However, this is the first study to describe the prevalence of pulmonary embolism at hospital admission.

Although a high prevalence of pulmonary embolism is reported in ICU (20.6-27\%) and at conventional wards (8.3\%) in patients with COVID-19, all these studies, the reported prevalence of pulmonary embolism is potentially biased since CTPA was only performed in COVID-19 patients with clinical aggravation and/or with clinical suspicion for pulmonary embolism and CTPA might not have been performed in intubated patients with a severe clinical course [1, 7-9]. In contrast, in our study, CTPA was performed routinely on admission regardless of whether pulmonary embolism was clinically suspected or not.

During follow up, no other pulmonary embolisms were diagnosed, suggesting that looking for pulmonary embolism at the time of admission, and using a prophylactic anticoagulant treatment in the absence of pulmonary embolism, is safe and prevents new pulmonary embolism during the in-hospital period and 3-month follow-up period.

One of the study limitations is the relatively small number of COVID-19 admitted patients. Indeed, our study started after the lockdown setting of our region that fortunately reduced the number of COVID-19 hospitalisation. Additionally, $21.5 \%$ of patients were excluded from the study, mostly due to contraindications to iodinated contrast injection or inability to understand verbal information about the study due to dementia. Lastly, there were protocol violations because D-dimer tests were not done in eight patients, two of whom had acute pulmonary embolism confirmed on CTPA. Large multicentre cohort studies are needed to confirm these data and explore the reasons why SARS-CoV-2 predisposes to VTE events, as no other respiratory viruses are known to do this [10-13].

In conclusion, this prospective study reported a high prevalence of pulmonary embolism in patients with COVID-19 at time of hospital admission. For this reason, we support CTPA exams on hospital admission in COVID-19 patients requiring supplemental oxygen, having high D-dimer concentrations or long history of COVID-19 symptoms, if no contraindicated.

Mitja Jevnikar ${ }^{1,2,3}$, Olivier Sanchez ${ }^{4,5,6}$, Richard Chocron $\oplus^{6,7}$, Marc Andronikof ${ }^{1,8}$, Maurice Raphael $^{1,9}$, Olivier Meyrignac ${ }^{1,10}$, Laure Fournier ${ }^{6,11}$, David Montani $\circledast^{1,2,3}$, Benjamin Planquette ${ }^{4,5,6}$, Mary Soudani ${ }^{1,12}$, Athénaïs Boucly ${ }^{1,2,3}$, Jeremie Pichon ${ }^{1,2,3}$, Mariana Preda ${ }^{1,2,3}$, Antoine Beurnier ${ }^{1,2,13}$, Sophie Bulifon ${ }^{1,2,3}$, Andrei Seferian ${ }^{1,2,3}$, Xavier Jaïs ${ }^{1,2,3}$, Olivier Sitbon $\oplus^{1,2,3}$, Laurent Savale $\oplus^{1,2,3}$, Marc Humbert $\oplus^{1,2,3}$ and Florence Parent ${ }^{1,2,3}$

${ }^{1}$ Université Paris-Saclay, Faculty of Medicine, Le Kremlin-Bicêtre, France. ${ }^{2}$ INSERM UMR_S 999, Le Kremlin-Bicêtre, France. ${ }^{3}$ AP-HP, Service de Pneumologie et soins intensifs respiratoires, Hôpital Bicêtre, Le Kremlin-Bicêtre, France. ${ }^{4} \mathrm{AP}-\mathrm{HP}$, Service de Pneumologie et Soins Intensifs, Hôpital Européen Georges Pompidou, Paris, France. ${ }^{5}$ INSERM UMR-S 1140; Paris, France and INNOVTE, StEtienne, France. ${ }^{6}$ Université Paris Descartes, Faculty of Medicine, Paris, France. ${ }^{7}$ AP-HP, Service d'Urgence, Hôpital Européen Georges Pompidou, Paris, France. ${ }^{8}$ AP-HP, Service d'Urgenc, Hôpital Antoine Béclère, Clamart, France. ${ }^{9} \mathrm{AP}-\mathrm{HP}$, Service d'Urgence, Hôpital Bicêtre, Le Kremlin-Bicêtre, France. ${ }^{10} \mathrm{AP}-\mathrm{HP}$, Service de Radiologie, Hôpital Bicêtre, Le Kremlin-Bicêtre, France. ${ }^{11} \mathrm{AP}-\mathrm{HP}$, Service de Radiologie, Hôpital Européen Georges Pompidou, Paris, France. ${ }^{12}$ AP-HP, Service de gériatrie, 
Hôpital Bicêtre, Le Kremlin-Bicêtre, France. ${ }^{13} \mathrm{AP}-\mathrm{HP}$, Service de physiologie et d'explorations fonctionnelles respiratoires (CRISALIS/F-CRIN network), Hôpital Bicêtre, Le Kremlin-Bicêtre, France.

Corresponding author: Florence Parent (florence.parent@aphp.fr)

Conflict of interest: M. Jevnikar has nothing to disclose. O. Sanchez has nothing to disclose. R. Chocron has nothing to disclose. M. Andronikof has nothing to disclose. M. Raphael has nothing to disclose. O. Meyrignac has nothing to disclose. L. Fournier has nothing to disclose. D. Montani has nothing to disclose. B. Planquette has nothing to disclose. M. Soudani has nothing to disclose. A. Boucly has nothing to disclose. J. Pichon has nothing to disclose. M. Preda has nothing to disclose. A. Beurnier has nothing to disclose. S. Bulifon has nothing to disclose. A. Seferian has nothing to disclose. X. Jaïs has nothing to disclose. O. Sitbon reports grants, personal fees and non-financial support from Actelion Pharmaceuticals and MSD, personal fees from Acceleron Pharmaceuticals, Gossamer Bio and Ferrer, grants and personal fees from Bayer, grants from GlaxoSmithKline, outside the submitted work. L. Savale has nothing to disclose. M. Humbert reports grants and personal fees from Actelion, personal fees from GSK and Merck, outside the submitted work. F. Parent has nothing to disclose.

\section{References}

$1 \quad$ Klok FA, Kruip MJHA, van der Meer NJM, et al. Incidence of thrombotic complications in critically ill ICU patients with COVID-19. Thromb Res 2020; 191: 145-147.

2 Helms J, Tacquard C, Severac F, et al. High risk of thrombosis in patients with severe SARS-CoV-2 infection: a multicenter prospective cohort study. Intensive Care Med 2020; 46: 1089-1098.

3 Frydman GH, Streiff MB, Connors JM, et al. The potential role of coagulation factor Xa in the pathophysiology of COVID-19: a role for anticoagulants as multimodal therapeutic agents. TH Open Companion J Thromb Haemost 2020; 4: e288-e299.

4 Zhou F, Yu T, Du R, et al. Clinical course and risk factors for mortality of adult inpatients with COVID-19 in Wuhan, China: a retrospective cohort study. Lancet 2020; 395: 1054-1062.

$5 \mathrm{Xu} \mathrm{X,} \mathrm{Yu} \mathrm{C,} \mathrm{Qu} \mathrm{J,} \mathrm{et} \mathrm{al.} \mathrm{Imaging} \mathrm{and} \mathrm{clinical} \mathrm{features} \mathrm{of} \mathrm{patients} \mathrm{with} 2019$ novel coronavirus SARS-CoV-2. Eur J Nucl Med Mol Imaging 2020; 47: 1275-1280.

6 Revel M-P, Parkar AP, Prosch H, et al. COVID-19 patients and the radiology department - advice from the European Society of Radiology (ESR) and the European Society of Thoracic Imaging (ESTI). Eur Radiol 2020; 30: 4903-4909.

7 Poissy J, Goutay J, Caplan M, et al. Pulmonary embolism in patients with COVID-19: awareness of an increased prevalence. Circulation 2020; 142: 184-186.

8 Fauvel C, Weizman O, Trimaille A, et al. Pulmonary embolism in COVID-19 patients: a French multicentre cohort study. Eur Heart J 2020; 41: 3058-3068.

9 Lodigiani C, lapichino G, Carenzo L, et al. Venous and arterial thromboembolic complications in COVID-19 patients admitted to an academic hospital in Milan, Italy. Thromb Res 2020; 191: 9-14.

10 Varga Z, Flammer AJ, Steiger P, et al. Endothelial cell infection and endotheliitis in COVID-19. Lancet 2020; 395: 1417-1418.

11 Magro C, Mulvey JJ, Berlin D, et al. Complement associated microvascular injury and thrombosis in the pathogenesis of severe COVID-19 infection: a report of five cases. Transl Res J Lab Clin Med 2020; 220: 1-13.

12 Ackermann M, Verleden SE, Kuehnel M, et al. Pulmonary vascular endothelialitis, thrombosis, and angiogenesis in Covid-19. N Engl J Med 2020; 383: 120-128.

13 Bunce PE, High SM, Nadjafi M, et al. Pandemic H1N1 influenza infection and vascular thrombosis. Clin Infect Dis Off Publ Infect Dis Soc Am 2011; 52: e14-17. 